\title{
Spontaneous Spinal Epidural Hematoma Coexisting Guillan-Barré Syndrome in a Child: A Case Report
}

\author{
Chi Hyung Lee, Geun Sung Song, Young Ha Kim, Dong Wuk Son, Sang Weon Lee \\ Department of Neurosurgery, Pusan National University Yangsan Hospital, \\ Pusan National University School of Medicine, Yangsan, Korea
}

Spontaneous spinal epidural hematoma (SSEH) has been reported as a rare cause of spinal cord compression, especially in children. Clinical features are usually nonspecific, although cervicothoracic location of hematoma could be presented with progressive paraplegia. Guillian-Barré syndrome (GBS) is clinically defined as an acute peripheral neuropathy causing progressive limb weakness. Because SSEH and GBS have very similar signs and symptoms, SSEH could be misdiagnosed as GBS. Nevertheless, they can be presented together. We describe a rare case of SSEH coexisting with GBS.

Key Words: Pediatrics $\cdot$ Guillain-Barré syndrome $\cdot$ Spinal epidural hematoma

\section{INTRODUCTION}

Spontaneous spinal epidural hematoma (SSEH) is a very rare cause of spinal cord compression, especially in children. Although SSEH has been reported to occur in all age groups, it is most frequent after the fourth or fifth decade ${ }^{16}$. SSEH is most often defined as a hematoma occurring in the absence of any trauma or iatrogenic procedure ${ }^{3)}$, however, Gopalkrishnan et al.") suggest that it should be named when hematoma is of idiopathic origin. Clinical presentations of SSEH are miscellaneous and vague, therefore differential diagnosis is important, especially in child. Here, we present the first case of SSEH concomitant with GBS in a child.

\section{CASE REPORT}

A previously healthy 32-month-old male presented to the Emergency Department (ED) with a 1-week history of progressive paraparesis. The patient had a cough and fever for about 2 weeks prior to admission to Pusan National University Yangsan Hospital. Five days before admission, he had difficulty moving his legs spontaneously, 4 days before admission he could not

\footnotetext{
- Received: April 1, 2016 • Revised: July 18, 2016

- Accepted: July 22, 2016

Corresponding Author: Geun Sung Song

Department of Neurosurgery, Research Institute for Convergence of Biomedical Science and Technology, Pusan National University School of Medicine, 20 Geumo-ro, Mulgeum-eup, Yangsan 50612, Korea Tel: +82-55-360-2126, Fax: +82-55-360-2156

E-mail: gnsong@pusan.ac.kr

$\otimes$ This is an open access article distributed under the terms of the Creative Commons Attribution Non-Commercial License (http://creativecommons.org/licenses/by-nc/4.0/) which permits unrestricted non-commercial use, distribution, and reproduction in any medium, provided the original work is properly cited.
}

walk without mother's help. On initial evaluation in the ED, the patient was afebrile with normal blood pressure. His neurologic examination was significant for $1 / 5$ strength in his both leg and deep tendon reflexes were absent. Lumbar puncture was performed and cerebrospinal fluid (CSF) examination revealed increased level of protein $(544 \mathrm{mg} / \mathrm{dL})$ without any other abnormality. Electromyography showed decreased conduction velocity and amplitude on lower limb. Under diagnosis of
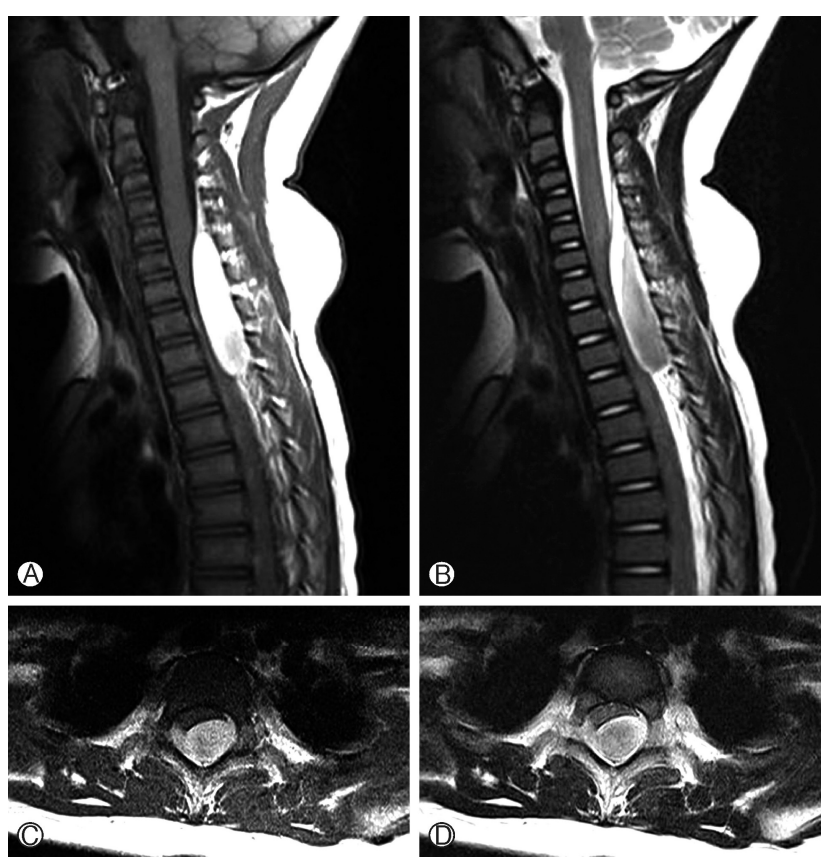

Fig. 1. Preoperative sagittal (A, B) and axial (C, D) magnetic resonance images showing a dorsal epidural hematoma extending from C4 to T3 level with cord compression. 
Guillain-Barré syndrome (GBS), he admitted to the pediatric department and received intravenous immunoglobulin (IVIG) therapy. Strength in his both leg was gradually improved (3/5) after four days of IVIG therapy, but he suddenly started to complain about neck pain. Spinal magnetic resonance image (MRI) demonstrated a dorsal epidural hematoma extending from C4 to T3 level with cord compression (Fig. 1). Conventional spinal angiography was performed to rule out spinal vascular abnormality and it revealed negative finding. His neurologic symptoms were neither improved nor progressed, and the patient was under febrile condition because of bronchopneumonia. Therefore, we decided to perform delayed removal of hematoma. Follow-up spinal MRI with contrast demonstrated enhancement of spinal nerve roots consistent with GBS and there was no significant interval change of the epidural hematoma (Fig. 2). C7 and T1 left hemilaminectomy and drainage of epidural hematoma was performed after 2 weeks of diagnosis of spinal epidural hematoma. Xanthochromic fluid was expelled and thecal sac expansion was identified in the surgical field. The postoperative course was uneventful and the patient regained strength, making a full recovery during 1 week of follow-up.

\section{DISCUSSION}

SSEH is a rare surgical emergency, comprising less than 1\% of spinal space-occupying lesions ${ }^{18)}$. Since the first case of SSEH was reported by Jackson in 1869, there have been numerous reports of such cases in the literature ${ }^{9)}$. Although SSEH has been reported to occur in all age groups, it is most frequent after the fourth or fifth decade, but is rare in children ${ }^{8,16}$.

Clinical presentations of SSEH are usually nonspecific and depend on the involved vertebral level, therefore the diagnosis of SSEH is difficult and differential diagnosis is important. There are several reports of SSEH mimicking meningitis, subarachnoid hemorrhage, GBS and even stroke ${ }^{5,10,13,15)}$. Although
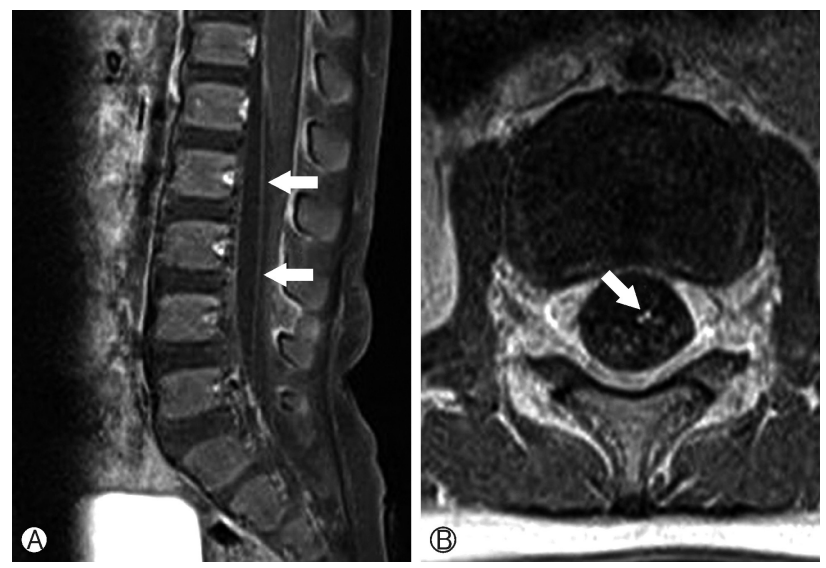

Fig. 2. Postcontrast sagittal T1-weighted image (A) and axial T1-weighted image (B) demonstrate abnormal enhancement of the nerve root (arrow). paraparesis or tetraparesis is a common symptom of cervical SSEH, hemiparesis can also be presented when the hematoma compresses the unilateral corticospinal tract. The SSEH located at the cervicothoracic junction, as in this case, may be presented with progressive paraplegia and neck pain. Cakir et al. ${ }^{5)}$ described a case of SSEH mimicking GBS in a child.

The most common pattern of GBS is the ascending paralysis, weakness beginning in the lower limbs first and migrating towards the trunk, and this is analogous to the symptoms of the SSEH located at the cervicothoracic junction. However, in the present case, there are several evidences of GBS; (1) albuminocytologic dissociation at CSF analysis, (2) decreased conduction velocity and amplitude on electromyography and (3) nerve root enhancement on enhanced spinal MRI. To the best of our knowledge, this is the first case report of SSEH concomitant with GBS.

The diagnosis of GBS is mainly based on clinical features and electrophysiologic and CSF studies. However, there are several reports of that enhanced spinal MR imaging can be used as a supplementary diagnostic modality in the diagnosis of GBS, when the clinical and electrophysiologic findings are equivocal $^{1,2}$. The presence of nerve root enhancement on enhanced spinal MRI can be the evidence of GBS, in the present case. Because nerve root generally does not take up much gadolinium on enhanced MRI, nerve root enhancement is thought to be secondary to a breakdown of the blood-nerve barrier.

Although GBS is a potentially life-threatening disease, even with the best treatment available, there are several reports of good prognosis in children, especially ${ }^{12,17}$. Salehiomran et al. ${ }^{17)}$ reported that 16 of 17 patients recovered completely (94.1\%). Immediate administration of IVIG after clinical suspicion is recommended, before irreversible nerve damage has $\operatorname{taken}^{19)}$.

Even though the pathogenesis of SSEH is remains unclear, 2 main mechanisms of SSEH have been suggested. The bleeding is mostly thought to be venous in origin. The vertebral venous plexus, a valveless low pressure system, is in continuity with the abdominal and thoracic venous system. Lack of valves in the epidural venous plexus makes the area especially vulnerable to any intrinsic change in pressure. The bleeding can occur after activities such as voiding, sneezing, bending, coughing, and coitus. Such activities of normal living can cause fluctuations in the intrathoracic and intra-abdominal pressures, with may cause reversal of blood flow resulting in rupture of a delicate vein in the valveless epidural plexus ${ }^{16}$. In the present case, repeated cough associated with upper respiratory tract infectio (URI) may be the cause of SSEH result from fluctuations of pressure in abdominal and thoracic venous system, as mentioned previously.

However, Beatty and Winston ${ }^{3)}$ suggest that the origin of SSEH is arterial origin, because the pressure of the epidural venous plexus is lower than the intrathecal pressure, hematomas usually form quickly, and arterial hypertension may sometimes coexist. Some suggest that these arteries are suscep- 
tible to abnormal stretching during symmetrical arm movements ${ }^{3}$.

SSEH can have catastrophic consequences including permanent neurologic deficit and even death, therefore urgent surgical decompression should be considered. Nevertheless, there have been a very few cases in which a good outcome after conservative mannagement ${ }^{4,6,11)}$. Preoperative neurologic deficit and the interval between symptom onset and surgical decompression are believed to be associated with the neurological outcome ${ }^{7,14)}$. Conservative management and delayed surgical decompression may be justified when there are minimal neurological findings or evidence of an early resolution of neurological deficits, as presented case.

\section{CONCLUSION}

SSEH is rare neurosurgical emergencies and require immediate diagnosis and treatment, because it can cause catastrophic consequences. However, clinical manifestations of the SSEH are nonspecific, so it seems to be misdiagnosed such as GBS, meningitis, and stroke. As seen in this case, SSEH can be concomitant with GBS and performing urgent spinal MRI should be considered when the patient has symptoms like neck pain and meningeal irritation sign.

\section{CONFLCT OF INTEREST}

No potential conflict of interest relevant to this article was reported.

\section{ACKNOWLEDGMENTS}

This work was supported by a 2-Year Research Grant of Pusan National University.

\section{REFERENCES}

1. Alkan O, Yildirim T, Tokmak N, Tan M: Spinal MRI findings of guillain-barré syndrome. J Radiol Case Rep 3:25-28, 2009

2. Baran GA, Sowell MK, Sharp GB, Glasier CM: MR findings in a child with Guillain-Barré syndrome. AJR Am J Roentgenol 161:161-163, 1993

3. Beatty RM, Winston KR: Spontaneous cervical epidural hematoma. A consideration of etiology. J Neurosurg 61:143-148, 1984

4. Borkar SA, Prasad GL, Satyarthee GD, Mahapatra AK: Sponta- neous spinal extradural hematoma in a child with hemophilia $\mathrm{B}$, surgery or medical management - A dilemma? J Pediatr Neurosci 6:131-133, 2011

5. Cakir E, Karaarslan G, Usul H, Baykal S, Kuzeyli K, Mungan I, et al: Clinical course of spontaneous spinal epidural haematoma mimicking Guillain-Barré syndrome in a child: a case report and literature review. Dev Med Child Neurol 46:838-842, 2004

6. Fedor M, Kim ES, Ding K, Muizelaar JP, Kim KD: Spontaneous spinal epidural hematoma: a retrospective study on prognostic factors and review of the literature. Korean J Spine 8:272-282, 2011

7. Gopalkrishnan CV, Dhakoji A, Nair S: Spontaneous cervical epidural hematoma of idiopathic etiology: case report and review of literature. J Spinal Cord Med 35:113-117, 2012

8. Groen RJ, van Alphen HA: Operative treatment of spontaneous spinal epidural hematomas: a study of the factors determining postoperative outcome. Neurosurgery 39:494-508, 1996

9. Jackson RR: Case of spinal apoplexy. Lancet 2:5-6, 1869

10. Jumani DB, Littlewood R, Iyer A, Fellows G, Healey A, Abernethy $\mathrm{L}$, et al: Spontaneous spinal epidural haematoma mimicking meningitis in a 2-year-old child: a case report and literature review. Childs Nerv Syst 29:1795-1798, 2013

11. Kim T, Lee CH, Hyun SJ, Yoon SH, Kim KJ, Kim HJ: Clinical outcomes of spontaneous spinal epidural hematoma: a comparative study between conservative and surgical treatment. J Korean Neurosurg Soc 52:523-527, 2012

12. Korinthenberg R, Schessl J, Kirschner J: Clinical presentation and course of childhood Guillain-Barré syndrome: a prospective multicentre study. Neuropediatrics 38:10-17, 2007

13. Labeodan OA: Spinal epidural haematoma mimicking spontaneous subarachnoid haemorrhage. Emerg Med J 22:606-607, 2005

14. Liao CC, Hsieh PC, Lin TK, Lin CL, Lo YL, Lee SC: Surgical treatment of spontaneous spinal epidural hematoma: a 5-year experience. J Neurosurg Spine 11:480-486, 2009

15. Matsumoto H, Miki T, Miyaji Y, Minami H, Masuda A, Tominaga $S$, et al: Spontaneous spinal epidural hematoma with hemiparesis mimicking acute cerebral infarction: two case reports. J Spinal Cord Med 35:262-266, 2012

16. Patel H, Boaz JC, Phillips JP, Garg BP: Spontaneous spinal epidural hematoma in children. Pediatr Neurol 19:302-307, 1998

17. Salehiomran MR, Nikkhah A, Mahdavi M: Prognosis of GuillainBarré syndrome in children. Iran J Child Neurol 10:38-41, 2016

18. Shin JJ, Kuh SU, Cho YE: Surgical management of spontaneous spinal epidural hematoma. Eur Spine J 15:998-1004, 2006

19. Van Doorn PA, Ruts L, Jacobs BC: Clinical features, pathogenesis, and treatment of Guillain-Barré syndrome. Lancet Neurol 7:939-950, 2008 